\title{
The Paradox Uncovering between Management and Engineering Concepts
}

\author{
Tianbo Zhang \\ Guangdong Industry Technical College \\ Guangdong,Guangzhou, 510300, China \\ e-mail:1985101083@gditc.edu.cn;QQ:723567650
}

\begin{abstract}
In terms of humanities and social system, the engineering exists among management, the management exists among engineering,"management" and "the engineering " seems to be nested within each other "paradox", but both are "thing(wu), things(shi), people, information "on the complex system. All management and engineering systems are the personnel systems,the personnel systems are complex systems,where the personnel system are the "giant complex adaptive intelligent systems" (GCAIS). The person's information, quantum, complexity, holographic, determines the nature of the "management" and " engineering" which is consistent. Management can be divided into "hard management" , "soft management" and "clever management "of three types; also be divided into littoscopic-management, microscopic- management, macroscopic-management, cosmoscopic-management and bulgoscopic- management ,of five levels. engineering having a certain hierarchical structure can also be given the same classification; it may be divided into tangible Engineering, invisible engineering, information engineering. The complementary principle of quantum mechanics reveals that the "management" and " engineering " are complementary in the personnel system.
\end{abstract}

Keywords- information complex holographic person; littoscopic-management(miaoguan guanli); ingenious management (qiao-guanli); wave-particle-information-awareness four images, (WPIAFI); ingenious engineering(qiao-gongcheng)

\section{INTRODUCTION}

In everyday life, literature and art, and even in the media parlance, Chinese context the concept of "management" and "engineering", its meaning seems very different from far away, but become increasingly integrated and nested within each other. In this paper, from the perspective of "information complex holographic person" hypothesis analyzed the connotation of the concept of "management "and "engineering". The person's information, quantum, complexity, holographic determines the nature of the "management" and " engineering" which is consistent. The complementary principle of quantum mechanics reveals the "management" and " engineering" are complementary in the personnel system.

\section{II. "INFORMATION COMPLEX HOLOGRAPHIC PERSON"}

\section{A. " Information Complex Holographic Person "} Definition

Biological person assumptions, economic person assumptions, rational person, cultural people assumptions, social person assumptions, complex person assumptions, information person hypothesis, self-realization person etc. these simple assumptions of human nature, can no longer meet the globalization, the needs of the global village, information society, network society, therefore, we must rethink the nature of the "people".The author has put forward that a man is composed by the tens of trillions of individual cells, ${ }^{[1]}$ person is a quantum, holographic, information, "giant complex adaptive intelligent systems "(Giant Complexity Adaptive Intelligent System, GCAIS referred to as: 格赛思) - referred to as "Information Complex Holographic Person "(abbreviated as:ICHP). ${ }^{[2]}$ Where there are human beings involved in active system, which are the giant complex adaptive intelligent systems, "management system" and "engineering system" are composed by the subject, object, media, and environmental and other components of the system, therefore, "management" and "engineering" are all " 格赛思" (GCAIS).

\section{B. The Characteristics of "People"}

In addition to the openness of the open complex giant system,the heterogeneity of the components, the level of the structure, the non-linearity of the relationship, dynamic behavior, both within and outside the uncertainty, "person" has also many of other characteristics, ${ }^{[3]}$ such as information feature, quantum feature, holographic features,etc.

The people 's quantum performance is that one can see the objective object,visual image of the object is received by the visual cells quantum fluctuations, in which network of nerve cells is seen in visual awareness. Light in the air, water and the propagation velocity of the glass is less than the speed of the vacuum, it is slowed down due to the interaction of the photon with substances,similarly, the light through the pupil and molten glass will slow down. Light quantum has wave-particle duality, any interaction with light can be considered to be quantized.

The human's hereditary indicates that gene cells is a holographic cell .Cloning technology illustrates the the hologram characteristics. A person is a "universe elements holographic point". 


\section{Man is a material and spiritual unity}

The author has suggested: consciousness produced in the nerve cells of the "Fluctuation-Particle-InformationAwareness Four images "(abbreviation: wave-particleinformation-awareness the four images, WPIAFI). ${ }^{[4]}$ This " wave-particle-information-awareness four images, (WPIAFI) " should be in the range of microscopic and miaoguan of brain tissue operation. Memory is brain thinking cells "Koch prominent fractal " of " Koch prominent". Limited brain capacity can accommodate unlimited "Koch prominent", so that the brain has unlimited memory. The microscopic nature of consciousness from the "WPIAFI",which makes that its material and spiritual is in one material and spiritual, they are the different forms of nerve cells or nerve particles.

\section{THE DEFINITION AND CLASSIFICATION OF MANAGEMENT CONCEPTS}

\section{A. The Definition of Management Concepts}

Western management mainly reflected in business management and administration. Definition of management has not yet unified, management was by some scholars grouped into variety:(1) Everyone is a manager, everybody is been managers; (2) Management is decision-making; (3) Management is to optimize the allocation of resources;(4) Management is a complex process of the survival and development; (5) Academician Liu Renhuai has thought that modern management is human activities, it is the service for the first type of activity.Management everywhere and at all times.This is called "Ubiquitous- management " (U-management).

《Zhouyi 》 makes that will days, earth and man be deemed to a opening up and has a holographic sexual of the non-linear the overall system to study, the body of person is closely related to the elephant of the universe, Any part of the body, a man is a microcosm of the world or that is a holographic point of the heavens and the earth. Managed objects can be the world, a country, a company of others and their own. Nature (the universe), and the world, country, home, personal five levels have isomorphism. Man is the nature of a hologram,the family is the holographic point of the country.

This paper argues that the definition of management is to:human society, the purpose of practical activities.Management can be seen as a complex, dynamic, unified whole and non-linear "work(zuoshi)" system. Regardless of human management, financial management, material management, or time, space, matter or mass (energy), information management, the starting point and destination point are "people", therefore, different assumptions of the "people" had a different management theory, the theory of human nature jungle "caused" the management theory jungle. In the network era of the information society, it has not then the "people" as a" whole person" or simply "rational person","person" shall be quantized complex holographic person. The corresponding assumptions with complex holographic theory should be "Chinese characteristics management theory".

\section{B. the classification of management concept}

As the object or scope of "management theory",it should extend to the depth and breadth in both directions, from the littoscopic-field to the bulgoscopic-field, the appropriate management can be referred to as: littoscopic-management, microscopic-management, macro-management, cosmoscopic management and bulgoscopic- management, a total of five levels.

"Littoscopic- management" is also called the" element management", the Saint and effort, its object is individual self-cultivation" of the management,such as the nature of things, extension of knowledge, Sacred Hearts, sincerity "and other psychological and knowledge cultivation.

"Microscopic-management" refers to the external performance of the individual self, which is outside the King of Kung $\mathrm{Fu}$, slim also is their own words and deeds think think the constraints, it is personally interacting with others in the manifestations.

"Macroscopic-management" may be understood on the management of the home or family, or the management of enterprises and administrative departments, which is the greatest volume of research management theory, including the management of specific types of engineering.

"Cosmoscopic management" refers to the management of a country or a province, city, or larger systems,the object of this management is complex temporal and spatial scales, composed of many heterogeneous elements.

"Bulgoscopic-management" corresponds to "the world", which is corresponding to the entire human activities, it is the social management.The society is formed by the people, the people around people and things "thing" together constitute the overall. The purpose of modern society refers to the management of the United Nations or between the countries.

The needs of the "people" can be divided into hard needs, soft needs and clever needs, three categories, appropriate management can be divided into three categories of "hard management", "software management" and "clever management"."Hard" corresponds to the "hard power" and "soft management" corresponds to the "soft power", "clever management" corresponds to the "smart power".

\section{THE DEFINITION OF ENGINEERING CONCEPTS, STRUCTURE AND CLASSIFICATION}

\section{A. The Definition of Engineering Concepts}

Although the concept of "engineering" is widely used, but it has not been recognized by the precise definition of "engineering". Different disciplines and different understanding of angles, "engineering" has been given a different meaning. It can be grouped into the following five forms: (1) "Engineering activities theory", the engineering is activities in the field of substance (Gaofeng Zhu, 2007); engineering is mass activities (Shanlin Yang, 2012).(2) "Labor process theory", the engineering is a manual process" (Ruiyu Yin, 2009). ${ }^{[5]}$ (3) "Engineering personnel systems",the engineering is personnel system.( Changfu $\mathrm{Xu}$, 2002).(4) "Engineering systems theory",engineering is a 
dynamic system of various factors (Yingluo Wang, 2008). ${ }^{[6]}$ (5) "Social formation theory",engineering concept is widely used in economic activities, social activities,which is to be called "social formation theory".Former Vice Premier Li Changchun said: "the implementation of Marxist theoretical research and construction project is a strategic engineering, life engineering, foundation engineering "( Changchun Li, 2012).

The above analysis, this paper argues can give the engineering a simple and broad definition: the engineering is the practical activities of human society, the purpose of the system.

The engineering involves elements of human, financial, material, matter, and information, to pay attention to the following three aspects: First of all, in addition to the purpose of the practice as a human population or individual activities,engineering is a human innovation activities. Innovation is the core elements of the engineering systems, innovation is the soul to improve project quality, innovation is the driving force behind the engineering. Secondly, people tend to focus only on the engineering group behavior while ignoring the individual practice to say, the practical activities of the individual may become a engineering, which is the socalled "littoscopic-engineering", which is the base of any other engineering yuan - yuan engineering. Third, it should be concerned about the use of specific projects after the completion of engineering structures "synergistic layer".

\section{B. Engineering Structure}

In general, almost all engineering activities, without exception, contain the following 10 basic elements: users, goals, resources, actors, methods and techniques, process, time and space, activities, environment, information. In all projects, there are things and things, persons and things, people and people, people and thing, things(shi) and things(shi) relationship, that there is a flow of people, material flow, energy flow, information flow. ${ }^{[7]}$ The framework of the engineering can be divided into the physical layer (base layer), the resource layer, implementation layer (operation level), the command layer (including feedback layer) and the efficiency layer (consumer level) five levels, abbreviated as WZZZZ or 1W4Z.

The physical layer is also called the base layer or the infrastructure layer. Resource layer refers to the sources of the various elements of the embodiment engineering. The execution level (operating level) is the concrete application of scientific knowledge, the type of technology or method skills of engineering practice activities, critical levels of the project can be completed successfully. The command layer (including feedback Layer) is the front end engineering decision-making, the interim implementation process command and adjusted post-consumer management, which is the brain or the soul of the project. The efficiency layer (consumer level) is simply more concerned about how to increase the effectiveness of the engineering to extend the life cycle of the engineering.

\section{The Classification of Engineering}

This paper argues that: due to the complexity and breadth of the engineering, according to the connotation and extension engineering and the size of the spatial and temporal scale, in general, the "engineering" can also be divided into: littoscopic-engineering, microscopicengineering, macroscopic-engineering, cosmoscopicengineering and bulgoscopic-engineering, five levels.

The "littoscopic- engineering" usually can be expressed the individual, work or practical activities involving macroscopic-engineering, microscopic-engineering elements, "littoscopic-engineering" is other engineering's primitives, it might be called "meta-engineering".

"Microscopic-engineering" refers to a grass-roots units or departments or several works carried out in a short period of time and less space, and it can be completed practical activities.

"Macroscopic-engineering" can be understood as it is involved in the industry, the engineering of a city or region, or scale is not too large, the involved small but far-reaching practical activities, such as the Guangzhou University City Engineering, etc.

"Cosmoscopic-engineering " refers to a country, a province, or involved in related projects within the scope of a few provinces, such as the Three Gorges Water Conservancy engineering, the National Games.

"Bulgoscopic- engineering" refers to the related to the engineering of all mankind and worldwide or several countries range.This "engineering" involves spatial and temporal scales, more complicated operation, had a big impact on the human and social, such as the implementation of the eEngineering of the United Nations or its specialized institutions, the Olympics, the World Health Organization, some are humanities and social works. Based on the different needs of the person, the corresponding engineering can also be divided into three classes of "hard engineering, soft engineering and clever engineering". According to the content or properties of engineering involved in host-guest, "engineering" can be divided into four types of tangible engineering, invisible engineering, personnel ngineering, information ngineering.In human economic and social life, the engineering concept is widely used, it can be said that engineering everywhere,This is called "ubiquitous engineering ".

\section{The ReleVance of Management AND ENGINEERING}

\section{A. Management and Engineering Essence of Consistency}

Based on the theory of complex systems and quantum "information complex holographic person" assumptions,management and engineering should be based on the establishment of the following three aspects. First, the basic assumptions of quantum "information complex holographic person "tells us that people is a" 格赛思" (GCAIS). Second, information technology, networking, holographic and intelligent are widely used in human science activities and social life.Third, as a natural part of the 
human, it is bound to a gradual transition from the "peopleoriented" and "human-centered" to "bio-based", "eco-centric ",to establish the concept of equality between man and nature.

Management exists in engineering; the engineering exists in management; management is part of the project implementation process.The engineering is the implementation of the management of organic whole, both nested within each other to form a mutually inclusive relationship, a "paradox" in the form of logic. Based on the quantum of the "information complex holographic person" perspective, the nature of the "management" and "engineering" is consistent.

\section{B. Management and Engineering Similarities or Isomorphism}

Engineering and management in the following three aspects are interlinked or are isomorphic. First, for "the cosmoscopic field" and "bulgoscopic-field", both engineering and management are all human beings involved in the natural and social material production and spiritual production practice activities, they all follow the "Wuli -Shili - Renli" methodology (WSR). Second, in the littoscopicfield, that is, in terms of the activity patterns of the human individual, engineering and management are also similar, that is quantized "information complex holographic person",deceptively simple in one fell move words and deeds, its essence is the GCAIS response to the external environment,which is very complex. This is also the engineering or management personnel on the basis of the activities. Third, in the area of collaborative innovation, whether it is engineering or management, collaborative innovation are the core elements , and collaborative innovation is the soul.

\section{Uncovering of "Paradox" between Management and Engineering Concept}

Management and engineering to form intertwined close relationship, in the final analysis, it is caused by the information, quantum and holographic. A single cell of the body contains all the information of the person,the overall is constituted by part, part of of the content includes the overall. Stem cells can be cloned from a living donor , which is holographic illustration. Engineering and management concept of "paradox" from human's information, quantum and holographic. The complementary principle of (complementary principle) of the quantum mechanics means that the mutual influence of the experimental apparatus for the observation target object. Management and engineering can be considered as two different sides of human social practice, they are complementary.

\section{CONCLUSION}

Human is logical starting point and ultimate goal of "the management" and " the engineering ". People are the core elements of the management activities and engineering activities. People's information, quantum and holographic leads to the complex system of the engineering and management systems,so that it becomes the with similar structure of "complex giant system",It makes the "management" and " engineering " nested within each other, each containing a "vicious circle" in the form of logic.

The Chinese context homeland, the governance of the country, and the world is the same, such as "moral" Chapter 54 "governing a large country is like cooking a small fish", it expressed its "engineering " holographic,cooking a small fish "and" governing a large country is isomorphic,cooking a small fish is the rule of the holographic point.Complementary principle is to understand the concept of "management" and "engineering " key to "paradox" phenomenon.

\section{ACKNOWLEDGMENT}

This work was supported by Ms. Qiuhong Li, and by Guangdong Province Education Science "12th Five-Year" Plan (No.:2012JK091), the Guangdong Higher Education Projects (No.:GJB125095).

[Author] Tianbo Zhang (1957-), male, Bozhou in Anhui, associate professor, corporate law consultant. mainly engaged in teaching, vocational education, management.

\section{REFERENCES}

[1] Tianbo Zhang, Haijuan Xu, Siyang Hu, Zhentai Lin.Based on the training base of the action system construction and exploring. [J].Research and Exploration in Laboratory,2011,30(08) : 437-441.

[2] Tianbo Zhang, Liliang Zhang. Public welfare of humanity under the framework of the assumption that occupational education[J]. Modern Enterprise Education, 2011, 391 (10) : 50-52.

[3] Tianbo Zhang,Youmie Luo. Faculty building in higher vocational college based on evolution of humanity hypothesis_-Guangdong Industry Technical College teachers in the cases. Education Teaching Forum, 2012, (37) : 224-227.

[4] Tianbo Zhang. Vocational education level Horizon the construction of modern vocational education system under the Exploration [C]. The third teaching management courses construction Conference Proceedings,2012:28-33.

[5] Ruiyu Yin. Engineering theory of evolution [J] Inception Engineering Research, Jan. 2009 (01) :75-82.

[6] Yingluo Wang.Contemporary concept of engineering and engineering education [J] China Engineering Science, 2008,10 (03) :17-20

[7] Tianbo Zhang. School productive training base construction [J]. Laboratory Research and Exploration, 2010,29 (08) :355-357. 